\title{
Root exudates and their molecular interactions with rhizospheric microbes
}

\begin{abstract}
Biologically important plant-microbe interactions are mediated by a wide array of signal compounds rhizodeposited from both plant and microbial species. Root exudates are some of the potentially important low molecular weight compounds secreted from plant roots. They are involved in building a network of biointeractions through several physical, chemical, or biological interactions. Application of bioinoculums has significantly improved growth parameters and yield of many economically valued crops. Root exudates mediate the plantmicrobe interactions by colonizing the roots and promoting root growth. Also, root exudates improve chemical and physical characteristics of the rhizospheric soil. Some of the beneficial plant-microbe associations include nitrogen fixation by rhizobium, symbiotic biointeractions with AM (arbuscular mycorrhizal) fungi, and PGPR (plant-growth-promoting Rhizobacteria). These interactions improve plant growth and quality, stress tolerance, and plant defense responses. Root exudates constitute a wide variety of secondary metabolite constituents that help plants to guard against microbial infections, insects, or herbivore attack. Root exudates secreted by plants act as antimicrobial agents to curb various harmful rhizospheric pathogens. In this chapter, we provide a summary of literatures on the significance of plant-microbe interactions in the improvement of plant morphological and biochemical features. Further, detailed information on various types of root exudates and their role in mediating plantmicrobe interactions and possible exploration of root exudates as a novel antimicrobial compounds are also discussed.
\end{abstract}

Keyword: Soil microbes; PGPR; Mycorrhizae; Signal molecules; Antimicrobials 